\title{
The effect of antenatal care on use of institutional delivery service and postnatal care in Ethiopia: a systematic review and meta-analysis
}

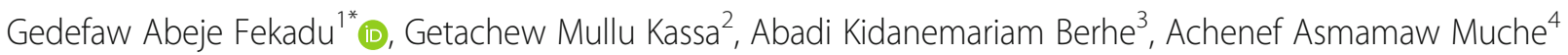
and Nuradin Abusha Katiso ${ }^{5}$

\begin{abstract}
Background: Although there are many initiatives to improve maternal health services use, utilization of health facility delivery and postnatal care services is low in Ethiopia. Current evidence at global level showed that antenatal care increases delivery and postnatal care services use. But previous studies in Ethiopia indicate contrasting results. Therefore, this meta-analysis was done to identify the effect of antenatal care on institutional delivery and postnatal care services use in Ethiopia.

Methods: Studies were searched from databases using keywords like place of birth, institutional delivery, and delivery by a skilled attendant, health facility delivery, delivery care, antenatal care, prenatal care and postnatal care and Ethiopia as search terms. The Joanna Briggs Critical Appraisal Tools and the Preferred Reporting Items for Systematic Review and Meta-Analyses were used for quality assessment and data extraction. Data analysis was done using STATA 14. Heterogeneity and publication bias were assessed using $P^{2}$ test statistic and Egger's test of significance. Forest plots were used to present the odds ratio (OR) with 95\% confidence interval (Cl).

Result: A total of 40 articles with a total sample size of 26,350 were included for this review and meta-analysis. Mothers who had attended one or more antenatal care visits were more likely $(O R=4.07: 95 \% \mathrm{Cl} 2.75,6.02)$ to deliver at health institutions compared to mothers who did not attend antenatal care. Similarly, mothers who reported antenatal care use were about four times more likely to attend postnatal care service (OR 4.11, 95\% Cl: 3.32, 5.09).

Conclusion: Women who attended antenatal care are more likely to deliver in health institutions and attend postnatal care. Therefore, the Ethiopian government and other stakeholders should design interventions that can increase antenatal care uptake since it has a multiplicative effect on health facility delivery and postnatal care services use. Further qualitative research is recommended to identify why the huge gap exists between antenatal care and institutional delivery and postnatal care services use in Ethiopia.
\end{abstract}

Keywords: Antenatal care, Postnatal care, Institutional delivery, Ethiopia, Meta-analysis

\footnotetext{
*Correspondence: abejegedefaw@gmail.com

'School of Public Health, College of Medicine and Health Sciences, Bahir Dar

University, P.O.Box 79, Bahir Dar, Ethiopia

Full list of author information is available at the end of the article
}

(c) The Author(s). 2018 Open Access This article is distributed under the terms of the Creative Commons Attribution 4.0 International License (http://creativecommons.org/licenses/by/4.0/), which permits unrestricted use, distribution, and reproduction in any medium, provided you give appropriate credit to the original author(s) and the source, provide a link to the Creative Commons license, and indicate if changes were made. The Creative Commons Public Domain Dedication waiver (http://creativecommons.org/publicdomain/zero/1.0/) applies to the data made available in this article, unless otherwise stated. 


\section{Background}

About 303,000 mothers died from pregnancy and childbirth related causes in 2015. Majority (99\%) of the deaths occurred in developing countries. Most of these deaths were from Sub-Sahara Africa [1, 2]. In Ethiopia, an estimated 11,000 mothers died due to pregnancy and childbirth related causes in 2015 [1, 3].

Globally, the major causes for maternal mortality are obstetric hemorrhage, hypertension, abortion, sepsis, HIV, preexisting medical disorders and other indirect causes like anemia [2, 4-6]. These are also causes of death for Ethiopian mothers [3, 7-10]. Most causes of maternal and child deaths are preventable or treatable with proven, cost-effective interventions [11-17]. A study conducted in India showed that $90 \%$ of maternal deaths would have been prevented if immediate decisions and appropriate care had been given at the time of delivery [18]. Provision of effective delivery care can prevent 113,000 maternal deaths annually [19].

Antenatal, delivery and postnatal care are among the key health sector interventions for maternal and child survival [20-28]. Many studies identified that antenatal care interventions reduce maternal and child mortalities and morbidities [29-34]. Institutional delivery can reduce the risk of neonatal mortality by $29 \%$ in low and middle-income countries [35, 36]. A study done in Southern and central India showed that increased institutional delivery is associated with decrease in stillbirth and perinatal mortality [37]. Similarly, skilled attendant at delivery can prevent and treat life-threatening conditions that may occur at the time of delivery [38-41]. Postnatal care is also a crucial time to tackle most causes of maternal and child mortality [42-45].

The Ethiopian government developed many strategies and programs to improve maternal and child health. For example, all maternal health services are provided free in Ethiopia [45-48]. The Health Extension program is another strategy which brought a tangible effect on family health $[48,49]$. The Ethiopian government set an ambitious plan to increase four or more ANC visits, delivery and postnatal care services use to 95, 90, and $95 \%$ respectively at the end of 2020 although the current level of these services use is low $[50,51]$.

Antenatal care is an opportunity to promote mothers to use other maternal health services $[34,45,52,53]$. Women who attended ANC are expected to use health facility delivery and attend postnatal care services. Yet, the situation is different in Ethiopia. According to the 2016 Ethiopian demographic and health survey, the proportion of women who attended ANC, health facility delivery and postnatal care is low compared to the national targets. Moreover, the proportion of mothers who delivered at health institutions (26\%) and attended postnatal care $(17 \%)$ is much lower than those who attended ANC $(64 \%)[50,51]$. Therefore, this review and meta-analysis were done to identify the effect of ANC on institutional delivery and postnatal care services use in Ethiopia. The result of this review and meta-analysis will help to identify whether antenatal care attendance has an effect on health facility delivery and postnatal care services use in Ethiopia.

\section{Methods}

\section{Search strategy}

We used the EndNote software and searched databases to retrieve studies for this review and meta-analysis. The search terms used were: place of birth, institutional delivery, delivery by a skilled attendant, health facility delivery, delivery care, antenatal care, prenatal care and postnatal care and Ethiopia. The main databases searched were PUBMED, MEDLINE, Google Scholar, web of science and African journal online (AJOL). After identifying the key literatures, their references were screened to retrieve additional articles.

\section{Evaluation of evidence}

To evaluate the quality of the papers, the Joanna Briggs Critical Appraisal Tools for review and meta-analysis was used. The AACODS (Authority, Accuracy, Coverage, Objectivity, Date, and Significance) was used to evaluate the quality of the articles $[54,55]$.

\section{Inclusion criteria}

The following criteria were used to include studies in this meta-analysis.

- Design: studies with all study design

- Publication status: both published and unpublished reports

- Language: literatures reported or published in English

- Publications or report year: papers published or reported up to September 05, 2017

- Place of study: studies that were conducted in Ethiopia regardless of the study setting (communitybased or institution based).

- Outcome reported: studies that reported the study outcomes (ANC and institutional delivery or ANC and postnatal care) or both

\section{Data abstraction}

This review was conducted from July 15 to September 05 , 2017. The review followed the Preferred Reporting Items for Systematic Review and Meta-Analyses (PRISMA) flow chart to identify and select relevant studies for this analysis. Initially, duplicated retrievals were removed. Then, studies whose titles were irrelevant for this study were excluded. After that, the abstracts were assessed and screened based on the exposure and outcome variables. At this stage, studies that were not relevant for this analysis were excluded. 
For the remaining articles, the full text was assessed. The eligibility of these articles was assessed based on the pre-set inclusion criteria. When articles did not have adequate data, corresponding authors of the research articles were contacted. All authors conducted the review independently and an agreement was reached through discussion when needed.

\section{Heterogeneity and publication bias}

Heterogeneity among the included studies was checked by using $I^{2}$ test statistic [56]. Heterogeneity was declared at $p \leq 0.05$. Publication bias was also checked by using Egger's test. A $p$-value of less than 0.05 was used to declare statistical significance of publication bias [57]. For studies which showed the presence of publication bias, the Duval and Tweedie nonparametric trim and fill analysis was conducted to account for the publication bias [58].

\section{Data analysis}

The analysis to identify the effect of ANC visits on institutional delivery service use was divided into two parts. The first analysis was to identify the effect of one or more ANC visits on institutional delivery service use and the second was an analysis of the effect of four or more ANC visits on institutional delivery service use.

Data were extracted from each study using data abstraction format prepared on Microsoft Excel. Then, the data were exported to STATA 14 for meta-analysis.

\section{Results}

\section{Description of the studies}

A total of 1236 records related to the review topics were identified. Ninety articles were removed because they were duplicates. Another 1139 articles were removed from the list after screening their title and abstracts. Then, full article review and screening was done for 59 studies. From these, a total of 20 articles were excluded for not reporting one or more of the outcome variable. Finally, 40 studies were included in the analysis (Fig. 1, Tables 1, 2 and 3).

The studies were conducted from 2011 to 2017. Most of the studies were from the four major regions of Ethiopia, 11 from Oromia, 17 from Amhara, 4 from Tigray and 5 from South Nations, nationalities and people's regional state. The sample size of the included studies ranged from 281 to 3472 participants. In terms of study design, all except three were cross-sectional (Tables 1, 2 and 3).

\section{Effect of ANC on institutional delivery service use}

A total of 30 studies with 26,350 sample size were included to estimate the effect of ANC on institutional delivery service use. The study populations for all the 30 studies were reproductive-age women who were pregnant or had given birth within 5 years of the survey. The studies were conducted from 2004 to 2016 (Table 1). Three of

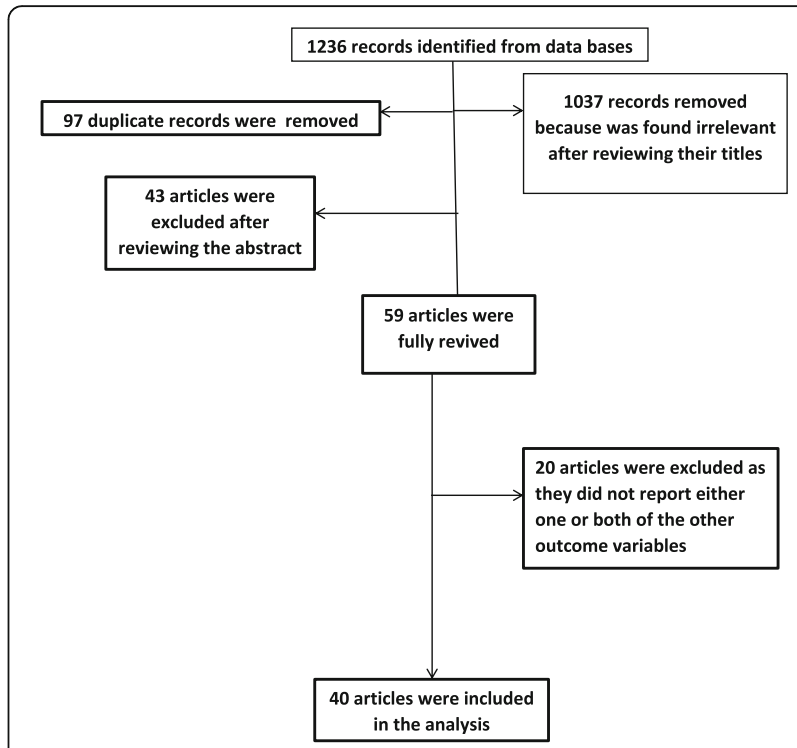

Fig. 1 Diagrammatic presentation of the procedure for selection of studies included to study the effect of antenatal care on institutional delivery service use and postnatal care in Ethiopia

the studies were case-control [59-61] and the remaining 27 studies were community-based cross-sectional or follow up studies [62-88].

This analysis identified that mothers who had one or more antenatal care visits were about four times more likely $(\mathrm{OR}=4.07: 95 \% \mathrm{CI} 2.75,6.02)$ to deliver at health facilities compared to mothers who had not attended ANC (Fig. 2).

Additionally, ten articles were included to assess the effect of four or more ANC visits on institutional delivery service use. The studies were conducted from 2013 to 2017. The total number of women included in this analysis was 8524 . Two of the studies were case-control $[59,60]$ and the other eight were cross-sectional studies [64, 67, $76,86,89-92]$. The sample size of the studies ranged from 320 to 3472 . The studies included in this subgroup analysis showed high heterogeneity $\left(I^{2}=87.8, \quad P \leq 0.001\right)$ but non-significant publication bias (Egger's test $=0.780$ ). Using the random effect model analysis, women who had four or more ANC visits were 4.38 times more likely to deliver in health facilities compared to women who reported fewer ANC visits (OR 4.38, 95\% CI: 2.96, 6. 48) (Fig. 3).

\section{Effect of ANC on postnatal care service use}

Six articles with a total sample size of 4047 women were included in this analysis. All except one (institution based) were community-based cross-sectional studies [79, 93-97]. There was no statistically significant heterogeneity and publication bias among the studies $\left(I^{2}=14.7, P=0.320\right.$ and Egger's test $=0.231$, respectively). The analysis indicated that mothers who attended ANC were about four times 
Table 1 Characteristics of studies included to study the effect of ANC visit on institutional delivery service use in Ethiopia

\begin{tabular}{|c|c|c|c|c|c|c|c|c|}
\hline \multirow[t]{2}{*}{ S.No- } & \multirow[t]{2}{*}{ Author and Year } & \multirow[t]{2}{*}{ Study area } & \multirow[t]{2}{*}{ Study period } & \multirow[t]{2}{*}{ Study design } & \multirow[t]{2}{*}{ Sample size } & \multirow[t]{2}{*}{ ANC attendance } & \multicolumn{2}{|c|}{ Institutional delivery } \\
\hline & & & & & & & Yes & No \\
\hline \multirow[t]{2}{*}{1} & \multirow[t]{2}{*}{ Tekelab et al., 2015 [62] } & \multirow{2}{*}{$\begin{array}{l}\text { East Wollega, } \\
\text { Oromia }\end{array}$} & \multirow[t]{2}{*}{ January, 2015} & \multirow[t]{2}{*}{ CB cross sectional } & \multirow[t]{2}{*}{801} & Yes & 240 & 254 \\
\hline & & & & & & No & 77 & 277 \\
\hline \multirow[t]{2}{*}{2} & \multirow[t]{2}{*}{ Tsegay et al., 2013 [63] } & \multirow{2}{*}{$\begin{array}{l}\text { Samri-Saharity } \\
\text { District, Tigray }\end{array}$} & \multirow[t]{2}{*}{ Not reported } & \multirow[t]{2}{*}{ CB cross sectional } & \multirow[t]{2}{*}{1115} & Yes & 39 & 563 \\
\hline & & & & & & No & 7 & 504 \\
\hline \multirow[t]{2}{*}{3} & Hailu et al., 2014 [64] & Tsegedie District, & November 2012 & CB cross sectional & 485 & Yes & 124 & 140 \\
\hline & & & to June 2013 & & & No & 29 & 192 \\
\hline 4 & Feyissa et al., 2014 [59] & East Wollega, & September to & Unmatched case & 320 & Yes & 73 & 168 \\
\hline & & & & & & No & 7 & 72 \\
\hline 5 & Mengesha et al., 2013 [60] & Dabat District, & October 2009 & Nested case & 1065 & Yes & 213 & 852 \\
\hline & & Amhara & to August, 2012 & control & & No & 152 & 159 \\
\hline 6 & Abebe et al., 2012 [61] & Bahir Dar Special & July, 2010 & Unmatched case & 324 & Yes & 205 & 57 \\
\hline & & & & & & No & 11 & 51 \\
\hline 7 & Abeje et al., 2014 [65] & Bahir Dar city & Jun to July, 2012 & CB cross sectional & 484 & Yes & 359 & 54 \\
\hline & & adminıstratıon, & & & & No & 20 & 14 \\
\hline 8 & Asres et al., 2015 [66] & Sheka zone, SNNP & February to March, & CB cross sectional & 554 & Yes & 319 & 126 \\
\hline & & & & & & No & 13 & 96 \\
\hline 9 & Odo et al., 2014 [67] & Goba town, Oromia & April, 2013 & CB cross sectional & 580 & Yes & 247 & 231 \\
\hline & & & & & & No & 17 & 67 \\
\hline 10 & Amano, 2012 [68] & Munisa Woreda, & April, 2011 & CB cross sectional & 855 & Yes & 74 & 223 \\
\hline & & & & & & No & 31 & 527 \\
\hline 11 & Teferra et al., 2012 [69] & Sekela District, & August, 2010 & CB cross sectional & 371 & Yes & 42 & 3 \\
\hline & & Amnara & & & & No & 206 & 120 \\
\hline 12 & Worku et al., 2013 [70] & North Gondar Zone, & January to March, 2012 & CB cross sectional & 1668 & Yes & 103 & 58 \\
\hline & & Amhara & & & & No & 145 & 170 \\
\hline 13 & Bayu et al., 2015 [71] & Southern Zone, & January to August & CB follow up & 522 & Yes & 263 & 82 \\
\hline & & ligray & & & & No & 68 & 52 \\
\hline 14 & Melaku et al., 2014 [72] & Kilite Awulalo, & September 2009 to & CB, longitudinal & 2361 & Yes & 536 & 1270 \\
\hline & & Tigray & August 2012 & & & No & 106 & 449 \\
\hline 15 & Abera et al., 2011 [73] & Arsi Zone, Oromia & February to March, & CB cross sectional & 1089 & Yes & 162 & 482 \\
\hline & & & & & & No & 14 & 416 \\
\hline 16 & Tura G, 2008 [74] & Metekel Zone, B/ & January to March, & CB cross sectional & 1060 & Yes & 108 & 409 \\
\hline & & Gumuz & 2001 & & & No & 17 & 504 \\
\hline 17 & Nigussie et al., 2004 [75] & North Gondar & November to & CB cross sectional & 1248 & Yes & 147 & 421 \\
\hline & & & December, 2002 & & & No & 21 & 653 \\
\hline 18 & Tura et al., 2014 [76] & Jimma Zone, & September 2012- & CB follow up study & 3472 & Yes & 954 & 1680 \\
\hline & & & & & & No & 110 & 728 \\
\hline 19 & Arba et al., 2016 [77] & Wolayta \& Dawuro & February to March, & CB cross sectional & 1000 & Yes & 326 & 435 \\
\hline & & & & & & No & 33 & 163 \\
\hline 20 & Bayu et al., 2015 [78] & Debremarkos town, & January to July, 2012 & $C B$, follow up & 422 & Yes & 232 & 116 \\
\hline & & & & & & No & 13 & 31 \\
\hline 21 & Darega et al., 2016 [79] & Abuna Gindeberet & March, 2013 & CB cross sectional & 703 & Yes & 98 & 481 \\
\hline & & & & & & No & 3 & 121 \\
\hline 22 & Demilew et al., 2016 [80] & Dangila district, & February, 2015 & CB cross sectional & 780 & Yes & 134 & 377 \\
\hline & & & & & & No & 6 & 246 \\
\hline 23 & Fikre and Demissie, 2012 [81] & Dodota District, & January, 2011 & CB cross sectional & 506 & Yes & 75 & 340 \\
\hline
\end{tabular}


Table 1 Characteristics of studies included to study the effect of ANC visit on institutional delivery service use in Ethiopia (Continued)

\begin{tabular}{|c|c|c|c|c|c|c|c|c|}
\hline \multirow[t]{2}{*}{ S.No } & \multirow[t]{2}{*}{ Author and Year } & \multirow[t]{2}{*}{ Study area } & \multirow[t]{2}{*}{ Study period } & \multirow[t]{2}{*}{ Study design } & \multirow[t]{2}{*}{ Sample size } & \multirow[t]{2}{*}{ ANC attendance } & \multicolumn{2}{|c|}{ Institutional delivery } \\
\hline & & & & & & & Yes & No \\
\hline & & Oromia & & & & No & 17 & 74 \\
\hline \multirow[t]{2}{*}{24} & \multirow[t]{2}{*}{ Habte and Demissie, 2015 [82] } & \multirow{2}{*}{$\begin{array}{l}\text { Cheha District, } \\
\text { SNNPR }\end{array}$} & \multirow{2}{*}{$\begin{array}{l}\text { December } 2012 \\
\text { to January } 2013\end{array}$} & \multirow[t]{2}{*}{ CB cross sectional } & \multirow[t]{2}{*}{845} & Yes & 251 & 483 \\
\hline & & & & & & No & 2 & 80 \\
\hline \multirow[t]{2}{*}{25} & \multirow[t]{2}{*}{ Kebede et al., 2013 [83] } & \multirow[t]{2}{*}{ Chilga, Amhara } & \multirow[t]{2}{*}{ March to June 2012} & \multirow[t]{2}{*}{ CB cross sectional } & \multirow[t]{2}{*}{475} & Yes & 54 & 218 \\
\hline & & & & & & No & 19 & 184 \\
\hline \multirow[t]{2}{*}{26} & \multirow[t]{2}{*}{ Kenea and Jisha, 2017 [84] } & \multirow{2}{*}{$\begin{array}{l}\text { Dale Wabera } \\
\text { District, Oromia }\end{array}$} & \multirow[t]{2}{*}{2014} & \multirow[t]{2}{*}{ CB cross sectional } & \multirow[t]{2}{*}{588} & Yes & 215 & 185 \\
\hline & & & & & & No & 45 & 122 \\
\hline \multirow[t]{2}{*}{27} & \multirow[t]{2}{*}{ Kidanu et al., 2017 [85] } & \multirow{2}{*}{$\begin{array}{l}\text { Dembecha District, } \\
\text { Amhara }\end{array}$} & \multirow[t]{2}{*}{ March, 2015} & \multirow[t]{2}{*}{ CB cross sectional } & \multirow[t]{2}{*}{700} & Yes & 6 & 45 \\
\hline & & & & & & No & 223 & 400 \\
\hline \multirow[t]{2}{*}{28} & \multirow[t]{2}{*}{ Tadele \& Lamaro, 2017 [86] } & \multirow[t]{2}{*}{ Bench Maji, SNNPRS } & \multirow[t]{2}{*}{ September, 2015} & CB cross sectional & 800 & Yes & 574 & 109 \\
\hline & & & & & & No & 25 & 57 \\
\hline 29 & Wako \& Kassa, 2017 [87] & Liben District, & June, 2015 & CB cross sectional & 876 & Yes & 76 & 444 \\
\hline & & Uromıa & & & & No & 34 & 237 \\
\hline 30 & Yigezu and Kitila, 2015 [88] & Jimma town, & February to & CB cross sectional & 281 & Yes & 165 & 63 \\
\hline & & Uromia & April, 2014 & & & No & 18 & 31 \\
\hline
\end{tabular}

CB Community based

Table 2 Characteristics of studies included to study the effect of number of ANC visits on institutional delivery service use in Ethiopia

\begin{tabular}{|c|c|c|c|c|c|c|c|c|}
\hline \multirow[t]{2}{*}{ S. No } & \multirow[t]{2}{*}{ Author and year } & \multirow[t]{2}{*}{ Study area } & \multirow[t]{2}{*}{ Study period } & \multirow[t]{2}{*}{ Study design } & \multirow[t]{2}{*}{ Sample size } & \multirow[t]{2}{*}{ Number of ANC visits } & \multicolumn{2}{|c|}{ Institutional delivery } \\
\hline & & & & & & & Yes & No \\
\hline \multirow[t]{2}{*}{1} & \multirow[t]{2}{*}{ Hailu et al., 2014 [64] } & \multirow{2}{*}{$\begin{array}{l}\text { Tsegedie District, } \\
\text { Tigray }\end{array}$} & \multirow{2}{*}{$\begin{array}{l}\text { November } 2012 \\
\text { to June } 2013\end{array}$} & \multirow{2}{*}{$\begin{array}{l}\text { CB cross } \\
\text { sectional }\end{array}$} & \multirow[t]{2}{*}{485} & $\geq 4^{+}$ & 29 & 14 \\
\hline & & & & & & $<4$ & 102 & 126 \\
\hline \multirow[t]{2}{*}{2} & \multirow[t]{2}{*}{ Feyissa et al., 2013 [59] } & \multirow{2}{*}{$\begin{array}{l}\text { East Wollega, } \\
\text { Oromia }\end{array}$} & \multirow{2}{*}{$\begin{array}{l}\text { September to } \\
\text { October } 2013\end{array}$} & \multirow{2}{*}{$\begin{array}{l}\text { Unmatched case } \\
\text { control }\end{array}$} & \multirow[t]{2}{*}{320} & $\geq 4^{+}$ & 48 & 79 \\
\hline & & & & & & $<4$ & 25 & 89 \\
\hline \multirow[t]{2}{*}{3} & \multirow{2}{*}{$\begin{array}{l}\text { Mengesha et al., } \\
2013[60]\end{array}$} & \multirow{2}{*}{$\begin{array}{l}\text { Dabat District, } \\
\text { Amhara }\end{array}$} & \multirow{2}{*}{$\begin{array}{l}\text { October } 2009 \\
\text { to August } 2012\end{array}$} & \multirow{2}{*}{$\begin{array}{l}\text { Nested case } \\
\text { control }\end{array}$} & \multirow[t]{2}{*}{1065} & $\geq 4^{+}$ & 152 & 159 \\
\hline & & & & & & $<4$ & 61 & 693 \\
\hline \multirow[t]{2}{*}{4} & \multirow[t]{2}{*}{ Odo et al., 2014 [67] } & \multirow{2}{*}{$\begin{array}{l}\text { Goba town, } \\
\text { Oromia }\end{array}$} & \multirow[t]{2}{*}{ April, 2013} & \multirow{2}{*}{$\begin{array}{l}\text { CB cross } \\
\text { sectional }\end{array}$} & \multirow[t]{2}{*}{580} & $\geq 4^{+}$ & 50 & 32 \\
\hline & & & & & & $<4$ & 196 & 200 \\
\hline \multirow[t]{2}{*}{5} & \multirow[t]{2}{*}{ Tura et al., 2014 [76] } & Jimma zone, & September 2012 & CB follow up & 3472 & $\geq 4^{+}$ & 633 & 595 \\
\hline & & Oromia & to April 2013 & study & & $<4$ & 321 & 1085 \\
\hline 6 & Alemayehu \& Mekonnen, & Ankasha Gagusa & February, 2014 & CB cross & 373 & $\geq 4^{+}$ & 23 & 22 \\
\hline & $2015[89]$ & woreda, Amhara & & sectional & & $<4$ & 41 & 199 \\
\hline 7 & Kasaye et al., 2017 [90] & Debremarkos town, & January, 2016 & CB cross & 518 & $\geq 4^{+}$ & 154 & 14 \\
\hline & & Amhara & & sectional & & $<4$ & 221 & 113 \\
\hline 8 & Tadele and Lamaro, & Bench Maji, & September, & CB cross & 800 & $\geq 4^{+}$ & 427 & 21 \\
\hline & $2017[86]$ & SNNPRS & 2015 & sectional & & $<4$ & 147 & 88 \\
\hline 9 & Desalegn et al., & Fogera District, & February - April, & CB cross & 412 & $\geq 4^{+}$ & 61 & 42 \\
\hline & 2014 [91] & Amhara & 2013 & sectional & & $<4$ & 65 & 231 \\
\hline 10 & Kibret, 2015 [92] & Gozamen District, & March to April, & CB cross & 499 & $\geq 4^{+}$ & 44 & 48 \\
\hline & & Amhara & 2014 & sectional & & $<4$ & 79 & 326 \\
\hline
\end{tabular}


Table 3 Characteristics of studies included to study the effect of antenatal care on post natal follow up in Ethiopia

\begin{tabular}{|c|c|c|c|c|c|c|c|c|}
\hline \multirow[t]{2}{*}{ S.No } & \multirow[t]{2}{*}{ Author and year } & \multirow[t]{2}{*}{ Study area } & \multirow[t]{2}{*}{ Study period } & \multirow{2}{*}{$\begin{array}{l}\text { Study design and } \\
\text { setting }\end{array}$} & \multirow[t]{2}{*}{ Sample size } & \multirow{2}{*}{$\begin{array}{l}\text { ANC } \\
\text { attendance }\end{array}$} & \multicolumn{2}{|c|}{ PNC attendance } \\
\hline & & & & & & & Yes & No \\
\hline \multirow[t]{2}{*}{1} & \multirow[t]{2}{*}{ Tesfahun et al., 2014 [93] } & \multirow{2}{*}{$\begin{array}{l}\text { Gondar Zuria district, } \\
\text { Amhara }\end{array}$} & \multirow{2}{*}{ April-August 2011} & \multirow{2}{*}{$\begin{array}{l}\text { Community based cross } \\
\text { sectional }\end{array}$} & \multirow[t]{2}{*}{836} & Yes & 550 & 155 \\
\hline & & & & & & No & 59 & 56 \\
\hline \multirow[t]{2}{*}{2} & \multirow[t]{2}{*}{ Darega et al., 2016 [79] } & \multirow{2}{*}{$\begin{array}{l}\text { Abuna Gindeberet } \\
\text { District, Oromia }\end{array}$} & \multirow[t]{2}{*}{ March, 2013} & \multirow{2}{*}{$\begin{array}{l}\text { Community based cross } \\
\text { sectional }\end{array}$} & \multirow[t]{2}{*}{703} & Yes & 210 & 369 \\
\hline & & & & & & No & 13 & 111 \\
\hline \multirow[t]{2}{*}{3} & \multirow[t]{2}{*}{ Limenih et al., 2016 [94] } & \multirow{2}{*}{$\begin{array}{l}\text { Debremarkos town, } \\
\text { Amhara }\end{array}$} & \multirow[t]{2}{*}{ November, 2014} & \multirow{2}{*}{$\begin{array}{l}\text { Community based cross } \\
\text { sectional }\end{array}$} & \multirow[t]{2}{*}{588} & Yes & 138 & 163 \\
\hline & & & & & & No & 59 & 228 \\
\hline \multirow[t]{2}{*}{4} & \multirow[t]{2}{*}{ Birhanu et al., 2016 [95] } & \multirow[t]{2}{*}{ Addis Ababa } & \multirow[t]{2}{*}{ April-May, 2016} & \multirow{2}{*}{$\begin{array}{l}\text { Institution based cross } \\
\text { sectional }\end{array}$} & \multirow[t]{2}{*}{422} & Yes & 273 & 139 \\
\hline & & & & & & No & 4 & 6 \\
\hline \multirow[t]{2}{*}{5} & \multirow[t]{2}{*}{ Hordofa et al., 2015 [96] } & \multirow{2}{*}{$\begin{array}{l}\text { Dembecha District, } \\
\text { Amhara }\end{array}$} & \multirow[t]{2}{*}{ July-August, 2013} & \multirow{2}{*}{$\begin{array}{l}\text { Community based cross } \\
\text { sectional }\end{array}$} & \multirow[t]{2}{*}{788} & Yes & 234 & 333 \\
\hline & & & & & & No & 22 & 147 \\
\hline 6 & Abosse et al. 2015, [97] & Hadya Zone, SNNPRS & January-February 2009 & Community based cross & 710 & Yes & 154 & 442 \\
\hline & & & & & & No & 3 & 92 \\
\hline
\end{tabular}

\section{Study}

ID

Abebe et al, 2012

Abeje et al, 2014

Abera et al, 2011

Amano etal, 2012

Arba et al, 2016

Asres 2015

Bayu et al, 2015

Bayu et al, 2015

Darega et al, 2016

Demlew et al, 2016

Feyissa etal, 2013

Fikir and Demssie, 2012

Habtie and Demssie, 2015

Hailu et al, 2014

Kebede et al, 2013

Kenea and Jisha, 2017

Kidanu et al, 2017

Melaku et al, 2014

Mengesha et al, 2013

Nigussie et al, 2004

Odo et al, 2014

Tadele and Lamaro, 2017

Teferra et al, 2012

Tekleab et al, 2015

Tsegay et al, 2013

Tura G, 2008

Tura et al, 2014

Wako and Kassa, 2017

Worku et al, 2013

Yigezu and Kitila, 2015

Overall (I-squared $=95.2 \%, p=0.000$ )

NOTE: Weights are from random effects analysis 0117
$\%$

OR $(95 \% \mathrm{Cl}) \quad$ Weight

$16.67(8.16,34.07) \quad 3.27$

$\begin{array}{ll}4.65(2.22,9.76) & 3.24\end{array}$

$9.99(5.70,17.51) \quad 3.41$

$5.64(3.61,8.83) \quad 3.50$

$\begin{array}{ll}3.70(2.48,5.53) & 3.53\end{array}$

$18.70(10.11,34.58) \quad 3.36$

$2.45(1.58,3.80) \quad 3.50$

$4.77(2.40,9.46) \quad 3.29$

$8.22(2.56,26.37) \quad 2.77$

$14.57(6.33,33.54) \quad 3.14$

$4.47(1.96,10.18) \quad 3.15$

$\begin{array}{ll}0.96(0.54,1.72) & 3.39\end{array}$

$20.79(5.07,85.26) \quad 2.48$

$5.86(3.70,9.28) \quad 3.49$

$2.40(1.37,4.19) \quad 3.41$

$3.15(2.12,4.67) \quad 3.53$

$0.24(0.10,0.57) \quad 3.11$

$1.79(1.41,2.26) \quad 3.61$

$0.26(0.20,0.34) \quad 3.60$

$10.86(6.76,17.43) \quad 3.48$

$4.21(2.40,7.39) \quad 3.41$

$\begin{array}{ll}12.01(7.19,20.05) & 3.45\end{array}$

$8.16(2.47,26.88) \quad 2.74$

$3.40(2.50,4.63) \quad 3.58$

$4.99(2.21,11.25) \quad 3.16$

$7.83(4.62,13.27) \quad 3.43$

$3.76(3.03,4.66) \quad 3.62$

$1.19(0.77,1.84) \quad 3.50$

$2.08(1.41,3.08) \quad 3.53$

$4.51(2.36,8.63)$

$4.07(2.75,6.02) \quad 100.00$

Fig. 2 Effect of any antenatal care visit on institutional delivery service use in Ethiopia 


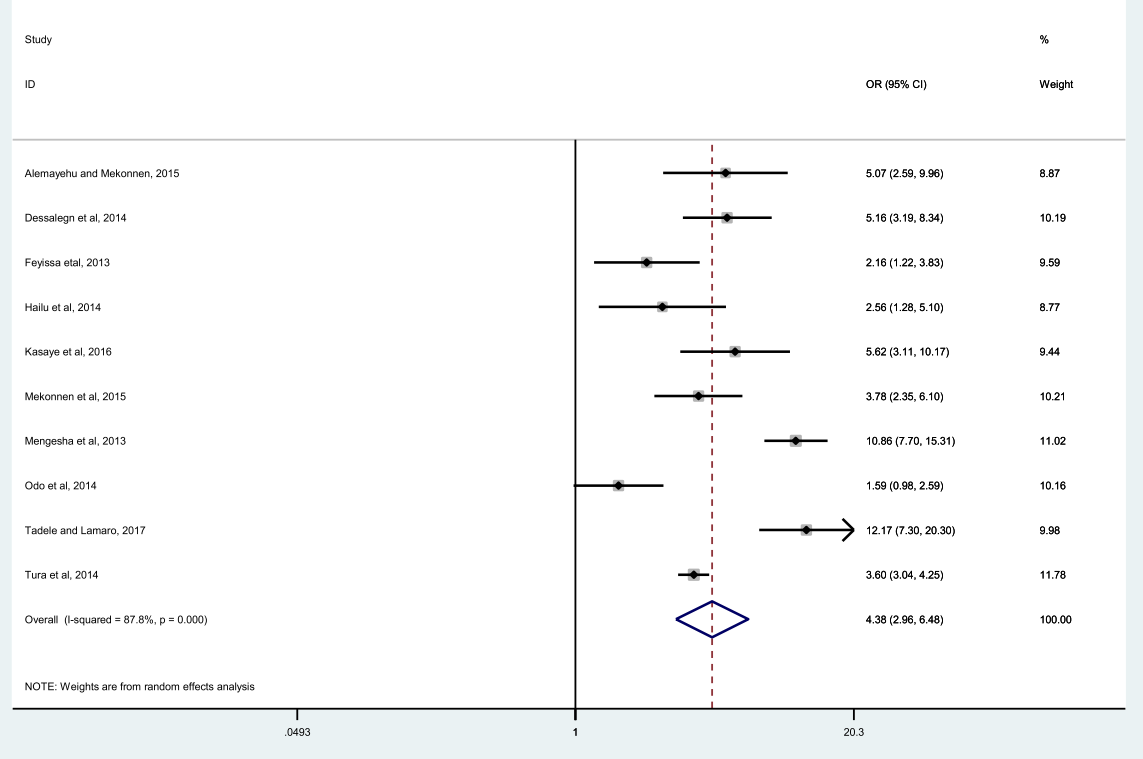

Fig. 3 Effect of four or more antenatal care visits on institutional delivery service use in Ethiopia

more likely to use postnatal care service (OR 4.11, 95\% CI: 3.32, 5.09) (Fig. 4).

\section{Discussion}

Antenatal care has been used as a strategy to reduce maternal and neonatal morbidities and mortalities. Various approaches and strategies have been implemented to improve the effectiveness of ANC in developing countries [52, 98, 99]. Currently, most developing countries including Ethiopia are using the focused ANC approach which was developed by WHO [100, 101].

This study identified that women who attended ANC were about four times more likely to use institutional delivery services. This finding was in line

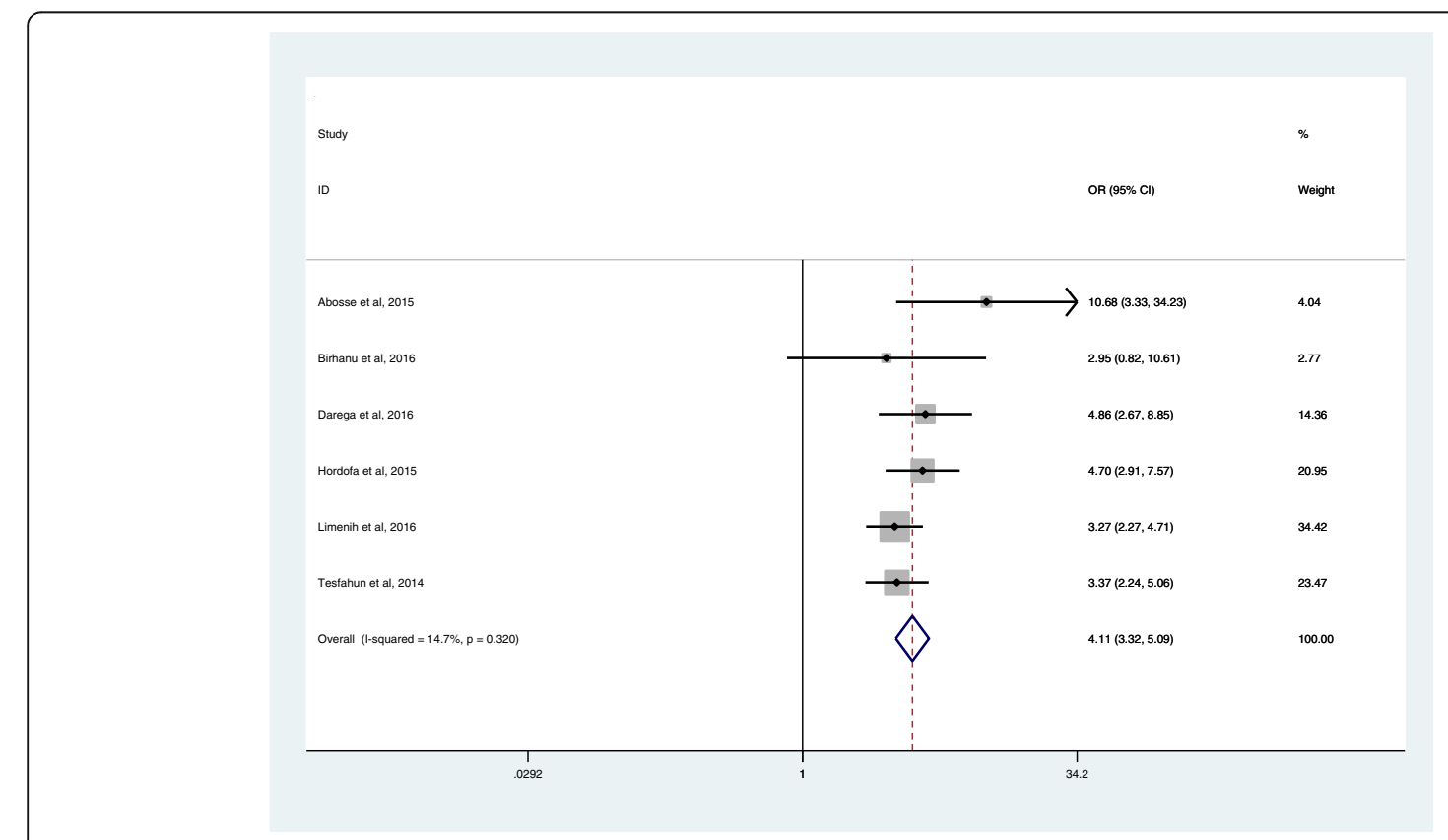

Fig. 4 Effect of antenatal care visit on postnatal care service use in Ethiopia 
with a meta-analyses conducted in Africa [102, 103] and DHS based data analysis in Nigeria [104]. The reason for this finding is that ANC is an opportunity for health promotion [105]. Therefore, women who attended ANC are more likely to have better information about benefits of institutional delivery service use and this may have impacted the subsequent health service use. Additionally, pregnant women attending ANC have the chance to acclimatize to the health facility environment. This may have helped them avoid unnecessary fear and stress related to institutional delivery service use. Furthermore, mothers who attended antenatal care are more likely to be better informed about danger signs and obstetric complications which may arise during labor and delivery. Antenatal care is also an opportunity for a pregnant woman to establish an informal forum which will help them to discuss and share information about their pregnancies and benefits of health facility delivery [46, 50, 57, 58].

The subgroup analysis showed four or more ANC visits had a similar effect on health facility delivery compared to fewer ANC visits. The reason for this may be that health professionals in developing countries provide all the information and health promotion activities needed for the mother on the first visit to avoid missed opportunities as the woman's return for the subsequent visits is not guaranteed [51, 105].

The current review also found that women who attended antenatal care were more likely to use postnatal care services. This finding is similar to studies conducted in Nigeria, Nepal, and Zambia [104, 106, 107]. It is theoretically plausible to think that mothers who attended ANC had received adequate counseling and information about postnatal care during the ANC session. Additionally, women may set birth plans in consultation with the ANC provider which in turn will increase delivery and postnatal service use [108].

This review had large sample size, which meant that it could detect the effect of ANC on institutional delivery and postnatal care services use. The analysis included all studies conducted in Ethiopia. But this meta-analysis does not address other factors that affect institutional delivery service use and postnatal care. In addition, this meta-analysis did not answer why institutional delivery and PNC services use remained low compared to ANC services use in Ethiopia. Evidence to identify the effect of ANC on PNC is limited. Therefore, we recommended further studies to identify the root cause for the huge difference in the proportion of women who attended ANC and PNC.

\section{Conclusion}

This review and meta-analysis revealed that mothers who attended ANC are more likely to use institutional delivery service and postnatal care. Mothers who attended ANC visits were more likely to deliver at health institutions. Similarly, women who attended ANC were more likely to attend postnatal care services. Therefore, the Ethiopian government and other stake holders need to exert collaborative effort to increase ANC service use since it has multiplicative on delivery and postnatal care services use.

\section{Abbreviations \\ AIDS: Acquired immune deficiency syndrome; ANC: Antenatal care; DHS: Demographic and health survey; EDHS: Ethiopian demographic and health survey; HIV: Human immune virus; OR: Odds ratio; SSA: Sub-Saharan Africa; UN: United Nations; UNICEF: United Nations International Children's Emergency fund; WHO: World Health Organization}

\section{Acknowledgements}

The authors would like to thank all the authors and publishers of the original studies.

\section{Authors' contributions}

GAF conceived the study. GAF and all others (GMK, AKB, AAM, and NAK) involved in the abstraction of the data, analysis, and writing of the study. The final manuscript was read and approved by all authors.

\section{Authors' information}

GAF is an assistant professor of Reproductive and Child health in Bahir Dar University, School of Public Health. GMK, AKB, AAM, and NAK are lecturers in Debremarkos University College of Health Sciences, Adigrat University,

College of Health Sciences, University of Gondar, Institute of Public Health and Wolaytasodo University College of health sciences respectively.

Ethics approval and consent to participate

Not applicable.

Consent for publication

Not applicable.

Competing interests

The authors declare that they have no competing interests

\section{Publisher's Note}

Springer Nature remains neutral with regard to jurisdictional claims in published maps and institutional affiliations.

\section{Author details}

${ }^{1}$ School of Public Health, College of Medicine and Health Sciences, Bahir Dar University, P.O.Box 79, Bahir Dar, Ethiopia. ${ }^{2}$ College of Health Sciences, Debre Markos University, Debre Markos, Ethiopia. ${ }^{3}$ College of Medicine and Health Science, Adigrat University, Adigrat, Tigray, Ethiopia. ${ }^{4}$ Department of Epidemiology and Biostatistics, Institute of public health, University of Gondar, Gondar, Ethiopia. ${ }^{5}$ Department of Nursing, College of Health Sciences and Medicine, Woliata Sodo University, Woliata Sodo, Ethiopia.

Received: 10 November 2017 Accepted: 9 July 2018

Published online: 24 July 2018

\section{References}

1. WHO U, UNFPA, World Bank Group and the United Nations Population Division. Trends in maternal mortality: 1990 to 2015: estimates by WHO, UNICEF, UNFPA, World Bank Group and the United Nations Population Division 2015.

2. Black RE, Levin C, Walker N, Chou D, Liu L, Temmerman M. Reproductive, maternal, newborn, and child health: key messages from disease control priorities 3rd edition. Lancet. 2016;388(10061):2811-24.

3. Tessema GA, Laurence CO, Melaku YA, Misganaw A, Woldie SA, Hiruye A, Amare AT, Lakew Y, Zeleke BM, Deribew A. Trends and causes of maternal mortality in Ethiopia during 1990-2013: findings from the global burden of diseases study 2013. BMC Public Health. 2017;17(1):160. 
4. Say L, Chou D, Gemmill A, Tunçalp Ö, Moller AB, Daniels J, Gülmezoglu AM, Temmerman M, Alkema L. Global causes of maternal death: a WHO systematic analysis. Lancet Glob Health. 2014;2(6):e323-e33.

5. Ronsmans CG, Wendy J. And lancet maternal survival series steering group. Maternal mortality: who, when, where, and why. Lancet. 2006;368(9542): $1189-200$.

6. Clark SL, Belfort MA, Dildy GA, Herbst MA, Meyers JA, Hankins GD. Maternal death in the 21st century: causes, prevention, and relationship to cesarean delivery. Am J Obstet Gynecol. 2008;199(1):36. e1-5.

7. Berhan Y, Berhan A. Causes of maternal mortality in Ethiopia: a significant decline in abortion related death. Ethiop J Health Sci. 2014;24:15-28.

8. Legesse T, Abdulahi M, Anteneh D. Trends and causes of maternal mortality in Jimma University specialized hospital, Southwest Ethiopia: a matched case-control study. Int J Womens Health. 2017;9:307.

9. Abdella A. Maternal mortality trend in Ethiopia. Ethiop J Health Dev. 2010; 24(1):115-22.

10. Gaym A. Maternal mortality studies in Ethiopia; magnitude, causes and trends. Ethiop Med J. 2009;47(2):95-108.

11. You D, Hug L, Ejdemyr S, Idele P, Hogan D, Mathers C, Gerland P, New JR, Alkema L. Global, regional, and national levels and trends in under-5 mortality between 1990 and 2015, with scenario-based projections to 2030: a systematic analysis by the UN Inter-agency Group for Child Mortality Estimation. Lancet. 2015;386(10010):2275-86.

12. Chou D, Daelmans B, Jolivet RR, Kinney M, Say L. Ending preventable maternal and newborn mortality and stillbirths. BMJ. 2015;351:h4255.

13. WHO. Strategies towards ending preventable maternal mortality (EPMM). 2015.

14. Bustreo F, Say L, Koblinsky M, Pullum TW, Temmerman M, Pablos-Méndez A. Ending preventable maternal deaths: the time is now. Lancet Glob Health. 2013;1(4):e176-e7.

15. Wani RJ, Chikhal P, Sonwalkar D. Maternal mortality: preventable tragedy. Bombay Hospital J. 2009:51(4):426-39.

16. Hezelgrave NL, Duffy SP, Shennan AH. Preventing the preventable: preeclampsia and global maternal mortality. Obstet Gynaecol Reprod Med. 2012;22(6):170-2.

17. Annan KA. Maternal health: investing in the lifeline of healthy societies \& economies: Africa progress panel; 2010.

18. Sundari KM, Priya RP. Maternal mortality: analysis of causes and preventable factors. Int J Reprod Contracept Obstet Gynecol. 2017;5(6):1719-21.

19. Bhutta ZA, Das JK, Bahl R, Lawn JE, Salam RA, Paul VK, et al. Can available interventions end preventable deaths in mothers, newborn babies, and stillbirths, and at what cost? Lancet. 2014;384(9940):347-70.

20. Prata N, Passano P, Sreenivas A, Gerdts CE. Maternal mortality in developing countries: challenges in scaling-up priority interventions. Women Health 2010;6(2):311-27.

21. Horton R. What will it take to stop maternal deaths? Lancet. 2009;374(9699): $1400-2$.

22. Hodgins S, Tielsch J, Rankin K, Robinson A, Kearns A, Caglia J. A new look at care in pregnancy: simple, effective interventions for neglected populations. PLoS One. 2016;11(8):e0160562.

23. Lassi ZS, Salam RA, Das JK, Bhutta ZA. Essential interventions for maternal, newborn and child health: background and methodology. Reprod Health. 2014;11(1):S1.

24. WHO. Three-year study identifies key interventions to reduce maternal, newborn and child deaths. Saudi Med J. 2012;33(1):105-7.

25. Adegoke A, Utz B, Msuya SE, Van Den Broek N. Skilled birth attendants: who is who? A descriptive study of definitions and roles from nine sub Saharan African countries. PLoS One. 2012;7(7):e40220.

26. Goldenberg RL, McClure EM. Maternal, fetal and neonatal mortality: lessons learned from historical changes in high income countries and their potential application to low-income countries. Matern Health Neonatol Perinatol. 2015;1(1):3.

27. WHO. Pregnancy, childbirth, postpartum and newborn care: a guide for essential practice. 3rd ed. Geneva: WHO, United Nations Population Fund, UNICEF; 2015.

28. Kerber KJ, de Graft-Johnson JE, Bhutta ZA, Okong P, Starrs A, Lawn JE. Continuum of care for maternal, newborn, and child health: from slogan to service delivery. Lancet. 2007;370(9595):1358-69.

29. Lucas AO, Stoll BJ, Bale JR. Improving birth outcomes: meeting the challenge in the developing world. Washington DC: National Academies Press; 2003.
30. Oyerinde K. Can antenatal care result in significant maternal mortality reduction in developing countries. J Community Med Health Educ. 2013;3(2):2-3.

31. Acharya S. How effective is antenatal care to promote maternal and neonatal health? Int J Gynecol Obstet. 1995;50:535-42.

32. Singh A, Pallikadavath S, Ram F, Alagarajan M. Do antenatal care interventions improve neonatal survival in India? Health Policy Plan. 2013; 29(7):842-8.

33. Carroli G, Rooney C, Villar J. How effective is antenatal care in preventing maternal mortality and serious morbidity? An overview of the evidence. Paediatr Perinat Epidemiol. 2001;15(s1):1-42.

34. Moos MK. Prenatal care: limitations and opportunities. J Obstet Gynecol Neonatal Nurs. 2006;35(2):278-85.

35. Tura G, Fantahun M, Worku A. The effect of health facility delivery on neonatal mortality: systematic review and meta-analysis. BMC Pregnancy Childbirth. 2013;13(1):18.

36. Fink G, Ross R, Hill K. Institutional deliveries weakly associated with improved neonatal survival in developing countries: evidence from 192 demographic and health surveys. Int J Epidemiol. 2015;44(6):1879-88.

37. Goudar SS, Goco N, Somannavar MS, Vernekar SS, Mallapur AA, Moore JL, et al. Institutional deliveries and perinatal and neonatal mortality in Southern and Central India. Reprod Health. 2015;12(2):S13.

38. Ronsmans C, Graham WJ, Group LMSSs. Maternal mortality: who, when, where, and why. Lancet. 2006;368(9542):1189-200.

39. Campbell OM, Graham WJ, Group LMSSs. Strategies for reducing maternal mortality: getting on with what works. Lancet. 2006;368(9543):1284-99.

40. Scott S, Ronsmans $C$. The relationship between birth with a health professional and maternal mortality in observational studies: a review of the literature. Tropical Med Int Health. 2009;14(12):1523-33.

41. Tanner J, AM AR, Candland T, Galdo V, Manang F, Trichler R, et al. Delivering the millennium development goals to reduce maternal and child mortality: a systematic review of impact evaluation evidence. 2014

42. WHO. Recommendations on postnatal care of the mother and newborn. Geneva: WHO; 2013. p. 2014

43. Sines E, Syed U, Wall S, Worley H. Postnatal care: a critical opportunity to save mothers and newborns, Policy Perspectives on Newborn Health; 2007. p. 1-7.

44. Lawn J. Saving mothers and newborn lives-the crucial first days after birth, The state of the world's children; 2009. p. 80-2.

45. Lawn J, Kerber K. Opportunities for Africa's newborns, vol. 246. Cape Town: The Partnership for Maternal, Newborn \& Child Health; 2006.

46. Zelelew $\mathrm{H}$. Health care financing reform in Ethiopia: improving quality and equity. 2014.

47. FDRE. National reproductive health strategy 2014-2018. In: Minstry of Health E, editor. Addis Ababa; 2014

48. Banteyerga $\mathrm{H}$. Ethiopia's health extension program: improving health through community involvement. MEDICC Rev. 2011;13(3):46-9.

49. Karim AM, Admassu K, Schellenberg J, Alemu H, Getachew N, Ameha A, et al. Effect of Ethiopia's health extension program on maternal and newborn health care practices in 101 rural districts: a dose-response study. PLoS One. 2013;8(6):e65160.

50. FDRE MoH. Health sector transformation plan. 2015.

51. CSA, ICF. Ethiopia Demographic and Health Survey 2016. Addis Ababa, and Rockville: CSA and ICF; 2016.

52. Stephenson P. Focused antenatal care: a better cheaper faster evidencebased approach. 2005

53. WHO. Antenatal care randomized trial: manual for the implementation of the new model. Geneva: World Health Organization; 2002. p. 37.

54. Munn Z, Moola S, Riitano D, Lisy K. The development of a critical appraisal tool for use in systematic reviews addressing questions of prevalence. Int $J$ Health Policy Manag. 2014;3(3):123.

55. Munn Z, Moola S, Lisy K, Riitano D. The Joanna Briggs institute reviewers' manual 2014, The systematic review of prevalence and incidence data. Adelaide: The Joanna Briggs Institute; 2014.

56. loannidis JP, Patsopoulos NA, Evangelou E. Uncertainty in heterogeneity estimates in meta-analyses. BMJ. 2007:335(7626):914.

57. Egger M, Smith GD, Schneider M, Minder C. Bias in meta-analysis detected by a simple, graphical test. BMJ. 1997;315(7109):629-34.

58. Duval S, Tweedie R. A nonparametric "trim and fill" method of accounting for publication bias in meta-analysis. J Am Stat Assoc. 2000;95(449):89-98.

59. Feyissa TR, Genemo GA. Determinants of institutional delivery among childbearing age women in western Ethiopia, 2013: unmatched case control study. PLoS One. 2014;9(5):e97194. 
60. Mengesha ZB, Biks GA, Ayele TA, Tessema GA, Koye DN. Determinants of skilled attendance for delivery in Northwest Ethiopia: a community based nested case control study. BMC Public Health. 2013;13(1):130.

61. Abebe F, Berhane Y, Girma B. Factors associated with home delivery in Bahirdar, Ethiopia: a case control study. BMC Res Notes. 2012;5(1):653.

62. Tekelab T, Yadecha B, Melka AS. Antenatal care and women's decision making power as determinants of institutional delivery in rural area of western Ethiopia. BMC Res Notes. 2015;8(1):769.

63. Tsegay Y, Gebrehiwot T, Goicolea I, Edin K, Lemma H, San Sebastian M. Determinants of antenatal and delivery care utilization in Tigray region, Ethiopia: a cross-sectional study. Int J Equity Health. 2013;12(1):30.

64. Hailu D, Berhe H. Determinants of institutional childbirth service utilisation among women of childbearing age in urban and rural areas of Tsegedie district, Ethiopia. Midwifery. 2014;30(11):1109-17.

65. Abeje G, Azage M, Setegn T. Factors associated with institutional delivery service utilization among mothers in Bahir Dar City administration, Amhara region: a community based cross sectional study. Reprod Health. 2014;11(1):22.

66. Asres A, Davey G. Factors associated with safe delivery service utilization among women in Sheka Zone, Southwest Ethiopia. Matern Child Health J. 2015;19(4):859-67.

67. Odo D, Shifti D. Institutional delivery service utilization and associated factors among child bearing age women in Goba Woreda, Ethiopia. J Gynecol Obstet. 2014;2(4):63-70.

68. Amano A, Gebeyehu A, Birhanu Z. Institutional delivery service utilization in Munisa Woreda, South East Ethiopia: a community based cross-sectional study. BMC Pregnancy Childbirth. 2012;12(1):105.

69. Teferra AS, Alemu FM, Woldeyohannes SM. Institutional delivery service utilization and associated factors among mothers who gave birth in the last 12 months in Sekela District, North West of Ethiopia: a community-based cross sectional study. BMC Pregnancy Childbirth. 2012;12(1):74.

70. Worku AG, Yalew AW, Afework MF. Maternal complications and women's behavior in seeking care from skilled providers in North Gondar, Ethiopia. PLoS One. 2013;8(3):e60171.

71. Bayu H, Fisseha G, Mulat A, Yitayih G, Wolday M. Missed opportunities for institutional delivery and associated factors among urban resident pregnant women in South Tigray Zone, Ethiopia: a community-based follow-up study. Glob Health Action. 2015;8(1):28082

72. Melaku YA, Weldearegawi B, Tesfay FH, Abera SF, Abraham L, Aregay A, et al. Poor linkages in maternal health care services-evidence on antenatal care and institutional delivery from a community-based longitudinal study in Tigray region, Ethiopia. BMC Pregnancy Childbirth. 2014;14(1):418.

73. Abera M, Belachew T. Predictors of safe delivery service utilization in Arsi Zone, South-East Ethiopia. Ethiop J Health Sci. 2011;21(3):95-106.

74. Tura G. Safe delivery service utilization in Metekel zone, northwest Ethiopia. Ethiop J Health Sci. 2008;18(1):213-22.

75. Nigussie M, Mariam DH, Mitike G. Assessment of safe delivery service utilization among women of childbearing age in North Gondar Zone, North West Ethiopia. Ethiop J Health Dev. 2004;18(3):145-52.

76. Tura G, Afework MF, Yalew AW. The effect of birth preparedness and complication readiness on skilled care use: a prospective follow-up study in Southwest Ethiopia. Reprod Health. 2014;11(1):60.

77. Arba MA, Darebo TD, Koyira MM. Institutional delivery service utilization among women from rural districts of Wolaita and Dawro Zones, Southern Ethiopia; a community based cross-sectional study. PLoS One. 2016;11(3): e0151082.

78. Bayu H, Adefris M, Amano A, Abuhay M. Pregnant women's preference and factors associated with institutional delivery service utilization in Debra Markos Town, North West Ethiopia: a community based follow up study. BMC Pregnancy Childbirth. 2015;15(1):15.

79. Darega B, Dida N, Tafese F, Ololo S. Institutional delivery and postnatal care services utilizations in Abuna Gindeberet District, West Shewa, Oromiya Region, Central Ethiopia: a community-based cross sectional study. BMC Pregnancy Childbirth. 2016;16(1):149.

80. Demilew YM, Gebregergs GB, Negusie AA. Factors associated with institutional delivery in Dangila District, North West Ethiopia: a crosssectional study. Afr Health Sci. 2016;16(1):10-7.

81. Fikre AA, Demissie M. Prevalence of institutional delivery and associated factors in Dodota Woreda (district), Oromia regional state, Ethiopia. Reprod Health. 2012;9(1):33.
82. Habte F, Demissie M. Magnitude and factors associated with institutional delivery service utilization among childbearing mothers in Cheha district, Gurage zone, SNNPR, Ethiopia: a community based cross sectional study. BMC Pregnancy Childbirth. 2015;15(1):299.

83. Kebede B, Gebeyehu A, Andargie G. Use of previous maternal health services has a limited role in reattendance for skilled institutional delivery: cross-sectional survey in Northwest Ethiopia. Int J Womens Health. 2013;5:79

84. Kenea D, Jisha H. Urban-rural disparity and determinants of delivery care utilization in Oromia region, Ethiopia: community-based cross-sectional study. Int J Nurs Pract. 2017:23(1):1-10.

85. Kidanu S, Degu G, Tiruye TY. Factors influencing institutional delivery service utilization in Dembecha district, Northwest Ethiopia: a community based cross sectional study. Reprod Health. 2017;14(1):98

86. Tadele N, Lamaro T. Utilization of institutional delivery service and associated factors in Bench Maji zone, Southwest Ethiopia: community based, cross sectional study. BMC Health Serv Res. 2017;17(1):101.

87. Wako WG, Kassa DH. Institutional delivery service utilization and associated factors among women of reproductive age in the mobile pastoral community of the Liban District in Guji zone, Oromia, Southern Ethiopia: a cross sectional study. BMC Pregnancy Childbirth. 2017;17(1):144

88. Yegezu R, Kitila S. Assessment of factors affecting choice of delivery place among pregnant women in Jimma Zone, South West Ethiopia: cross sectional study. J Womens Health Care. 2015;4(211):1-4.

89. Alemayehu M, Mekonnen W. The prevalence of skilled birth attendant utilization and its correlates in North West Ethiopia. Biomed Res Int. 2015;2015

90. Kasaye HK, Endale ZM, Gudayu TW, Desta MS. Home delivery among antenatal care booked women in their last pregnancy and associated factors: community-based cross sectional study in Debremarkos town, North West Ethiopia, January 2016. BMC Pregnancy Childbirth. 2017;17(1):225.

91. Desalegn E, Mekonnen A, Abeje G. Place of delivery after antenatal care: the case of Fogera district, Amhara region, North West, Ethiopia; 2013. J Gynecol Obstet. 2014;2(1):1-6.

92. Kibret GD. Prevalence and determinants of home birth after AnteNatal Care attendance in Gozamin District, Northwest Ethiopia. Health Sci J. 2015:9(69):1-7.

93. Tesfahun F, Worku W, Mazengiya F, Kifle M. Knowledge, perception and utilization of postnatal care of mothers in Gondar Zuria District, Ethiopia: a cross-sectional study. Matern Child Health J. 2014;18(10):2341-51.

94. Limenih MA, Endale ZM, Dachew BA. Postnatal care service utilization and associated factors among women who gave birth in the last 12 months prior to the study in Debre Markos Town, Northwestern Ethiopia: a communitybased cross-sectional study. Int J Reprod Med. 2016;2016:7095352.

95. Senait Berhanu Yeshi Asefa (RN B, MSc), Berhanu Wordofa Giru (BSC, MSC), School of nursing and midwifery AAU, Ethiopia. Prevalence of postnatal care utilization and associated factors among women who gave birth and attending immunization clinic in selected government health centers in Addis Ababa, Ethiopia. J Health Med Nurs 2016;26:2016.

96. Hordofa M, Almaw S, Berhanu M, Lemiso H. Postnatal care service utilization and associated factors among women in Dembecha District. Northwest Ethiopia. 2015;3(5):686-92.

97. Abosse Z, Woldie M, Ololo S. Magnitude and predictors of postnatal care utilization in Hadiya Zone, South Ethiopia. Int J Curr Res. 2015; 7(11):23176-82

98. Dujardin B, Clarysse G, Criel B, De Brouwere V, Wangata N. The strategy of risk approach in antenatal care: evaluation of the referral compliance. Soc Sci Med. 1995;40(4):529-35.

99. Yuster E. Rethinking the role of the risk approach and antenatal care in maternal mortality reduction. Int J Gynecol Obstet. 1995;50:S59-61.

100. Tunçalp Ö, Pena-Rosas JP, Lawrie T, Bucagu M, Oladapo OT, Portela A et al. WHO recommendations on antenatal care for a positive pregnancy experience-going beyond survival. BJOG Int J Obstet Gynaecol. 2017; 124(6):860-2.

101. WHO. Recommendations on antenatal care for a positive pregnancy experience. 2016.

102. Adjiwanou V, LeGrand T. Does antenatal care matter in the use of skilled birth attendance in rural Africa: a multi-country analysis. Soc Sci Med. 2013; 86:26-34.

103. Berhan Y, Berhan A. Antenatal care as a means of increasing birth in the health facility and reducing maternal mortality: a systematic review. Ethiop J Health Sci. 2014;24:93-104. 
104. Dahiru T, Oche OM. Determinants of antenatal care, institutional delivery and postnatal care services utilization in Nigeria. Pan Afr Med J. 2015;22(1):1-17.

105. Lawn J, Kerber K, Ou C, Yang H, Balinandi S, Sawadogo S, et al. Opportunities for Africas newborns: practical data policy and programmatic support for newborn care in Africa. J Virol Methods. 2007;144(1-2):109-14.

106. Khanal V, Adhikari M, Karkee R, Gavidia T. Factors associated with the utilisation of postnatal care services among the mothers of Nepal: analysis of Nepal demographic and health survey 2011. BMC Womens Health. 2014; 14(1):19.

107. Jacobs C, Moshabela M, Maswenyeho S, Lambo N, Michelo C. Predictors of antenatal care, skilled birth attendance, and postnatal care utilization among the remote and poorest rural communities of Zambia: a multilevel analysis. Front Public Health. 2017;5:11.

108. Magoma M, Requejo J, Campbell O, Cousens S, Merialdi M, Filippi V. The effectiveness of birth plans in increasing use of skilled care at delivery and postnatal care in rural Tanzania: a cluster randomised trial. Tropical Med Int Health. 2013;18(4):435-43.

Ready to submit your research? Choose BMC and benefit from:

- fast, convenient online submission

- thorough peer review by experienced researchers in your field

- rapid publication on acceptance

- support for research data, including large and complex data types

- gold Open Access which fosters wider collaboration and increased citations

- maximum visibility for your research: over $100 \mathrm{M}$ website views per year

At $\mathrm{BMC}$, research is always in progress.

Learn more biomedcentral.com/submissions 